\title{
Stem cell-based therapies threatened by the accumulation of $\mathrm{p} 53$ mutations
}

$c 4$

This study

... highlights

the need for

careful genetic

analyses of

stem cells

and their

differentiated

derivatives

before clinical
Human embryonic stem (ES) cells can self-renew indefinitely and give rise to virtually all cell types in the body. This makes them a valuable source of cells for regenerative therapies. ES cell-derived differentiated cells are being evaluated in clinical trials for their safety in therapeutic interventions for several diseases. McCarroll, Eggan and colleagues now report that human ES cells accumulate mutations in TP53 the gene that encodes the tumour suppressor $\mathrm{p} 53$ - which is mutated in $~ 50 \%$ of cancers.

Previous studies have shown that cultured human pluripotent stem cells (PSCs) can acquire aneuploidy and large copy number variations, and that these mutations can confer

a growth advantage to cells.

The authors set out to identify other mutations that could be acquired in culture, by sequencing all exons in the genome (exomes) of 140 independent cultured ES cell lines (114 lines maintained by the US National
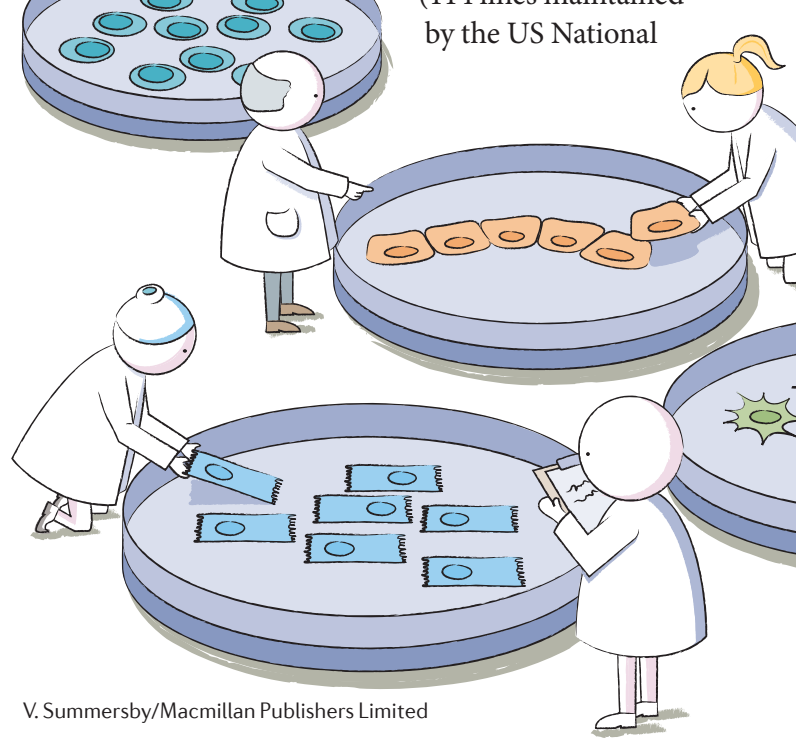

culture, and estimated that they

were present in a substantial

fraction (14-80\%) of cells in the affected cell lines. Analysis of cells from early culture

Institutes of Health and 26 lines prepared under good manufacturing practice conditions for potential clin ical use). They then used computational analyses to identify mutations that were present only in a subset of cells in each ES cell line, thus excluding inherited polymorphisms. With is approach, they identified 263 candidate mosaic variants, gene function.

The authors went on to characterize these 28 mutations and, strikingly, found that six of them were in TP53, which was also the only gene that was mutated more than once. The six mutations were identified in five unrelated cell lines. The six missense mutations (all involving a cytosine residue of a $\mathrm{CpG}$ dinucleotide) mapped to four residues in the DNAbinding domain of p53. Mutations at positions have been shown to wild-type 553 from binding to the promoters of its target genes, and are associated with a high risk of developing cancer.

The authors showed that all six passages confirmed that the TP53 mutations conferred selective advantage, with the proportion of mutant alleles increasing $~ 1.9$-fold per passage. a strong
This finding is consistent with previous reports that $\mathrm{p} 53$ loss promotes cell survival, proliferation and the reprogramming of somatic cells to pluripotency.

Lastly, an analysis of publically available RNA sequencing data from 117 human PSC lines (that are capable of differentiating into different cell types) revealed another eight missense mutations in TP53 that are distinct from the six identified in the present study, but which are also in the p53 DNA-binding domain. Some of these published studies used the same source cell line, indicating that the mutations also arose during cell culture.

This study indicates that cultured human PSCs have a high propensity to accumulate cancer-related mutations in TP53, with implications for their use in disease modelling and cell replacement therapies. It demonstrates the need to develop new culture conditions that could reduce the selective pressure for TP53 mutations to occur and, crucially, it highlights the need for careful genetic analyses of stem cells and their differentiated derivatives before clinical use. Importantly, the study also indicates that sequencing can be used to detect potentially harmful mutations and thus increase the safety of cell replacement therapies.

Kim Baumann

ORIGINAL ARTICLE Merkle, F. T., Ghosh S. et al. Human pluripotent stem cells recurrently acquire and expand dominant negative P53 mutations. Nature http://dx.doi.org/10.1038/nature22312 (2017)

FURTHER READING Trounson, A. \& DeWitt, N. D, Pluripotent stem cells progressing to the clinic. Nat. Rev. Mol. Cell Biol. 17, 194-200 (2016)| Avior, Y., Sagi, I. \& Benvenisty, N. Pluripotent stem cells in disease modelling and drug discovery. Nat. Rev. Mol. Cell Biol. 17, 170-182 (2016) 